\title{
MODELOS DE INVESTIGACIÓN OPERATIVA EN LA GESTIÓN DE RECURSOS HUMANOS
}

\author{
Carmela Velázquez Pino ${ }^{1}$ \& Esther Berger Vidal ${ }^{1}$ \& Inés Gambini López ${ }^{1}$ \\ Lucy de la Cruz Cuadros ${ }^{1}$
}

Resumen.- En las Facultades de la Universidad, como en toda organización, existen unidades organizacionales que tienen funciones relacionadas a la Gestión de Recursos Humanos, tales como la Dirección Administrativa, Escuelas y Departamentos Académicos e inclusive la Comisión de Evaluación y Perfeccionamiento Docente.

En el contexto descrito, nuestro interés está en desarrollar un estudio con los siguientes objetivos: Contar con un Sistema de Asignación de Carga a los Docentes de la Facultad de Ciencias Matemáticas, en particular en el Departamento Académico de Investigación Operativa, que garantizará una distribución objetiva e imparcial y permitirá la selección del docente idóneo para el dictado de un curso, con esta finalidad se ha formulado un modelo de Programación Lineal.

Se plantea además una cadena de Markov que traduce, en una matriz de probabilidades de transición, los cambios de categoría de los docentes nombrados de la Facultad de Ciencias Matemáticas entre procesos de Promoción Docente consecutivos.

Palabras claves: Cadena de Markov, Investigación Operativa, recursos humanos.

\section{OPERATIONAL RESEARCH MODELS IN HUMAN RESOURCES MANAGEMENT}

\begin{abstract}
In the University faculties, as in every organization, there are organizational entities that have functions related to human resource management, such as the administrative management, academic department, faculties, even the committee assessment and the theaching improvement.

In the context described above, our interest is to develope a study concerned with the following objectives: To have an assignment system charge of the professors of the Mathematic Science Faculty, in particular in the Operational Research deparment, which guarantees an objective and impartial distribution that will allow to select the suitable professor to teach a particular course, with this purpose, it has been formulated a linear program model.

Moreover, we set out a Markov's chain which translate in a transition probability matrix the changes of category of the permanent professor of the
\end{abstract}

\footnotetext{
${ }^{1}$ UNMSMS, Facultad de Ciencias Matemáticas.
} 
Mathematic Science Faculty among consecutives professor promotion procedure.

Key words: Markov's Chain, Operational Research, human resources.

\section{INTRODUCCIÓN:}

La aplicación de la Investigación Operativa en la Gestión de Recursos Humanos es, quizás, el área menos desarrollada en este campo, y es también la más difícil de promover. Esto se debe a que la dirección de personal de la organización debe responder adecuadamente a las siguientes demandas, mediante el uso apropiado del personal:

1. La organización requiere contar con trabajo efectivo y un rol estructural definido con el fin de producir resultados (bienes y servicios) en cantidad y calidad satisfactorias. Teoría de la Organización y Dirección.

2. Se hace necesario para la organización el uso eficiente de sus recursos. Ingeniería Industrial e Investigación Operativa.

3. El personal requiere satisfacer necesidades básicas y de actualización: Teoría del Comportamiento.

4. La sociedad requiere la producción y distribución de bienes y servicios compatibles con una postura ética respecto a los deberes, derechos y privilegios de sus ciudadanos. Ciencias Sociales - Ética.

El investigador de operaciones debe considerar todos estos aspectos o demandas en la formulación del problema de administración del potencial humano.

El objetivo de aplicar Investigación Operativa en la gestión de Recursos Humanos es asegurar, en cierto modo, un mejoramiento del personal por medio del apoyo a la toma de decisiones óptimas a la Dirección de Personal, cuyas funciones básicas son:

I. Reclutamiento, selección y socialización de personal.

II. Desarrollo: Proporcionando suministros para la Educación, Preparación y Desarrollo del Personal.

III. Distribución o Asignación: Asignando el mejor personal a tareas y roles. 
IV. Utilización: Determinando la estructura organizacional, patrones de flujo de información y comunicación, estilo de liderazgo y esquema de motivación.

V. Evaluación: Midiendo la contribución individual a la organización.

VI. Apoyo y Compensación: Determinando las recompensas económicas y no lucrativas necesarias para mantener cada contribución individual a la organización y la apropiada recompensa individual. Esta función incluye salarios y premios, beneficios, promociones, condiciones de trabajo, status, reconocimiento e incentivos sociales.

VII. La Investigación Operativa ofrece metodologías y técnicas para formular y resolver modelos que han sido utilizados en cada una de las áreas de decisión inmersas en las funciones básicas citadas. Entre ellos tenemos los modelos de transporte y asignación, de Programación Lineal, Programación de Metas, de Asignación secuencial, de Simulación y las cadenas de Markov.

La Investigación Operativa con su enfoque sistémico y metodologías también se aplica con propiedad en la Planificación de Recursos Humanos, proporcionando ratios para el análisis de los puestos de trabajo y de las cargas de trabajos.

\section{EL MODELO DE PROGRAMACIÓN LINEAL}

Variables de decisión:

Si se consideran $m$ docentes y $n$ cursos

$X_{i j}$ : Docente $i(i=1,2, \ldots, m)$, asignado al curso $j(j=1,2, \ldots, n)$

$X_{i j}=1$ si al Docente $i$ se le asigna el curso $j$

$X_{i j}=0$ si al docente $i$ no se asigna al curso $j$

Sean las ponderaciones siguientes:

- $P_{i j}=$ Prioridad del Docente $i$ por el curso $j$

$P i j=1,2,3$

- $A_{i j}=$ Categoría y Clase del Docente $i$ en el curso $j$

$A_{i j}=8 \leftrightarrow$ Principal DE y TC

$A_{i j}=7 \leftrightarrow$ Principal TP

$A_{i j}=6 \leftrightarrow$ Asociado DE y TC

$A_{i j}=5 \leftrightarrow$ Asociado TP 


$$
\begin{aligned}
& A_{i j}=4 \leftrightarrow \text { Auxiliar DE y TC } \\
& A_{i j}=3 \leftrightarrow \text { Auxiliar TP } \\
& A_{i j}=2 \leftrightarrow \text { Jefe de Prácticas DE y TC } \\
& A_{i j}=1 \leftrightarrow \text { J.P. TP }
\end{aligned}
$$

- $B_{i j}=$ Preparación del docente $i$ en el dictado del curso $j$.

$$
\begin{aligned}
B_{i j} & =1 \leftrightarrow \text { Deficiente } \\
B_{i j} & =2 \leftrightarrow \text { Regular } \\
B_{i j} & =3 \leftrightarrow \text { Bueno } \\
B_{i j} & =4 \leftrightarrow \text { Muy Bueno } \\
B_{i j} & =5 \leftrightarrow \text { Excelente } \\
\Rightarrow C_{i j} & =P_{i j}+A_{i j}+B_{i j}
\end{aligned}
$$

El Modelo de Asignación de Docentes a cursos es el Modelo de Programación Lineal siguiente:

$$
\begin{aligned}
& \text { máx } Z=\sum_{i=1}^{m} \sum_{j=1}^{n} C_{i j} X_{i j} \\
& \text { s.a: }
\end{aligned}
$$

(1) $\sum_{i=1}^{m} X_{i j}=1 ; j=1,2, \ldots, n$ cursos

(2) $\sum_{j=1}^{n} X_{i j} \leq 3 ; j=1,2, \ldots, m$ docentes

(3) $X_{i j}=0,1 ; \forall i, \forall j$

Se han tenido en cuenta las prioridades $P_{i j}$, porque en cada Departamento Académico, antes de la asignación de carga Docente, se le proporciona al docente un formato en el que consigna sus prioridades (1,2 ó 3) por determinados cursos.

En lo que se refiere a las ponderaciones $B_{i j}$ (preparación del docente $i$ en el curso j) puede ser obtenido a través de las encuestas a los alumnos que se deberían realizar semestre a semestre en la Facultad.

En el modelo general de Programación Lineal propuesto, en lo que respecta a las restricciones, se interpretan del modo siguiente:

(1) $\sum_{i=1}^{m} X_{i j}=1 ; j=1,2, \ldots, n$ cursos 
significa que se asignará solo un profesor $i$ a un curso $j$, el índice $j$ puede tomar hasta dos valores para referirse a un solo curso, si éste consiste de horas de Teoría, horas de Práctica - Laboratorio.

(2) $\sum_{j=1}^{n} X_{i j} \leq 3 ; j=1,2, \ldots, m$ docentes

A cada profesor se le asigna a lo más tres cursos diferentes.

Este modelo constituye un modelo general y un plan piloto, y el modelo que resulta de acuerdo a la realidad de cada Departamento, consiste de un gran número de variables y restricciones.

\section{CADENA DE MARKOV}

Se plantea una cadena de Markov que traduce, en una matriz de probabilidades de transición, los cambios de categoría de los docentes nombrados de la Facultad de Ciencias Matemáticas, entre dos procesos de Promoción Docente consecutivos, siendo el último el del año 2000. El modelo resultante es un indicador de cuál ha sido el movimiento de los docentes entre las categorías Principal, Asociado, Auxiliar y Jefe de Práctica, como consecuencia de estos Procesos de Evaluación, que permitirá hacer un estudio de las políticas de actualización y capacitación docentes, así como una revisión de los Reglamentos de Promoción Docente y Concurso Público, y el Reglamento que norma la justificación de horas lectivas y no lectivas de la tarea docente.

El modelo estocástico que se propone es el siguiente:

\section{CADENA DE MARKOV ASOCIADA AL CAMBIO DE CATEGORÍA DE LOS DOCENTES NOMBRADOS DE LA FACULTAD DE CIENCIAS MATEMÁTICAS: $1996-2000$}

\begin{tabular}{|l|ccccc|}
\hline & Principal & Asociado & Auxiliar & Jefe de Prácticas & Fuera \\
\hline P & 0.85 & 0 & 0 & 0 & 0.15 \\
AS & 0.21 & 0.47 & 0 & 0 & 0.32 \\
AUX & 0 & 0.24 & 0.36 & 0 & 0.40 \\
JP & 0 & 0 & 0.25 & 0.47 & 0.28 \\
F & 0 & 0 & 0 & 0 & 1 \\
\hline
\end{tabular}




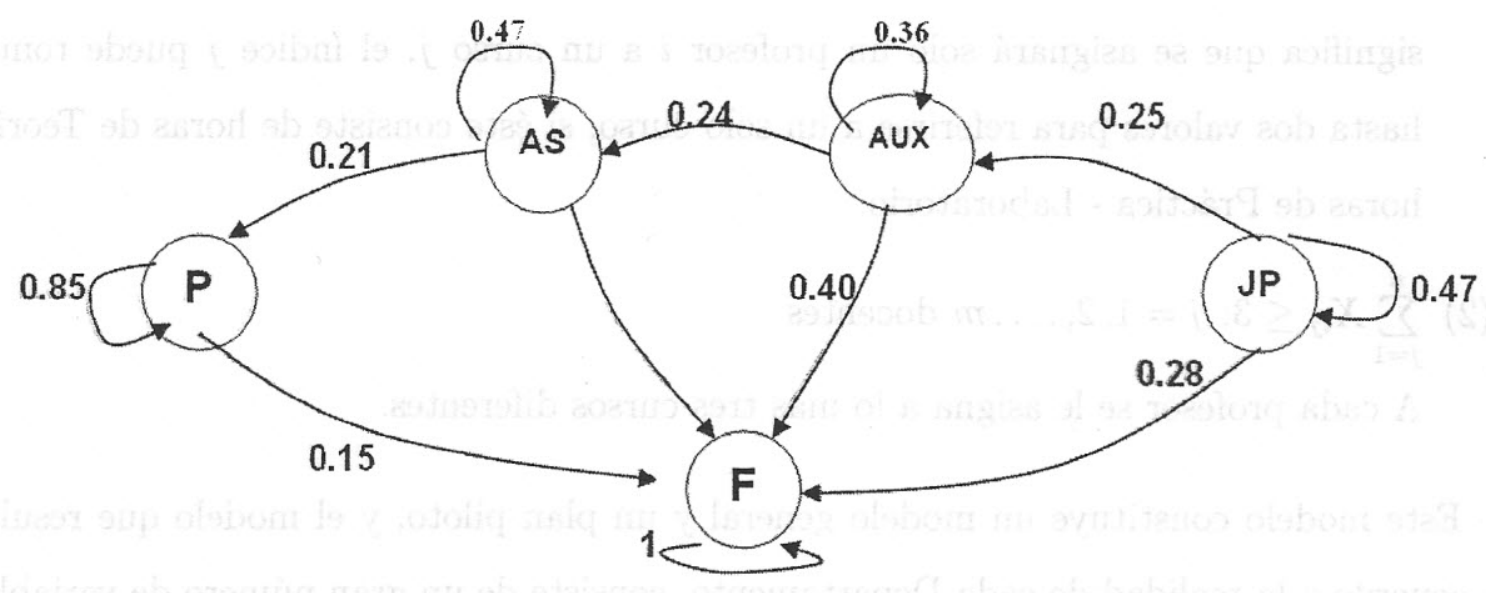

Los estados que se consideran están relacionados con las categorías Docentes:

$$
\begin{aligned}
\mathrm{P} & =\text { Principal } \\
\mathrm{As} & =\text { Asociado } \\
\text { Aux } & =\text { Auxiliar } \\
\mathrm{JP} & =\text { Jefe de Práctica } \\
\mathrm{F} & =\text { Fuera }
\end{aligned}
$$

En F = Fuera, se considera: (1) Los Profesores de Investigación Operativa y Computación que fueron trasladados en diciembre de 1996 al Departamento de Ciencias de la Computación, para la Escuela de Ingeniería de Sistemas creada en dicha fecha, y (2) los profesores cesantes.

La distribución de probabilidades de estado inicial es:

$$
\begin{aligned}
& P_{O}=[0,075,0,304,0,414,0,207,0] \\
& \begin{array}{ccccc}
\uparrow & \uparrow & \uparrow & \uparrow & \uparrow \\
\text { Princ. } & \text { Asoc. } & \text { Aux } & \text { J.P. } & \text { F }
\end{array}
\end{aligned}
$$

Año $\mathrm{O}=1996$

La cadena de markov resultante es una cadena de estados absorbentes y transitorios:

Fuera $=\mathrm{F}=$ Estado Absorbente

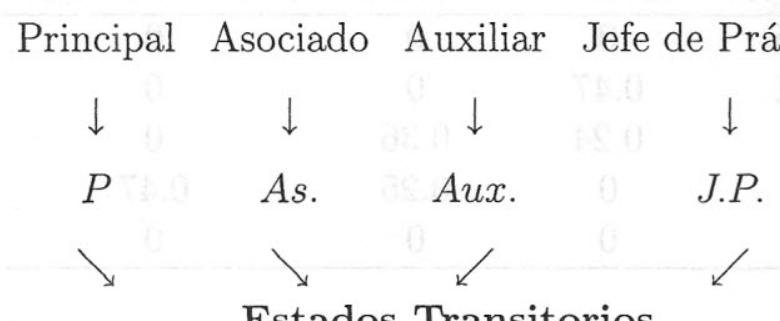

\section{Estados Transitorios}

El estudio realizado es original y es una investigación Básica, adaptativa, aplicativa y exploratoria, no tiene conexión a un Programa de Investigación en Desarrollo. 


\section{CONCLUSIONES:}

Formulación de un modelo general de asignación de cursos a los docentes del Departamento de Investigación Operativa.

- Construcción de una cadena de Markov para mostrar los cambios de categoría de los docentes de la Facultad de Ciencias Matemáticas como producto de los Procesos de Evaluación 1996 - 2000.

\section{REFERENCIAS}

[1] William B. Werethe, Jr. Y Keith Davis - Administración de Personal y Recursos Humanos (2000). Mc. Graw-Hill.

[2] R. Wayne Mondy y Robert M. Noe - Administración de Recursos Humanos (1997), Prentice-Hall Hispanoamerican, S.A.

[3] Buffa y Dyer - Ciencias de la Administración e Investigación de Operaciones (1983), Editorial Limusa.

[4] Winston, Wayne L. - Investigación de Operaciones (1994), Grupo Editorial Iberoamerica.

[5] G.D. Eppen, F.J. Gould, C.P. Schmidt, Jeffrey H. Moore - Investigación de Operaciones en la Ciencias Administrativa (2000), Prentice Hall Hispanoamericana, S.A.

[6] David R. Anderson, Dennis J. Sweeney y Thomas a. Williams - Métodos Cuantitativos para los negocios (1999), International Thomson Editores.

[7] Brtholomew. D.J. - Stochastic Models for Social Processes (1978), John Wiley and Sons.

[8] Lieberman, Gerald J. - Stochastic Models (1974), Mc Graw-Hill.

[9] Investigación Operativa - Revista de ALIO. 